examination and those with drug exposure $>5$ yrs were prioritised. An MDT pathway was established to manage anyone with signs of HCQ toxicity.

Results: 2,132 patients are prescribed $\mathrm{HCQ}$ in our county with population of 669,000 . 997 patients fell under our unit's remit. 136 patients ( $82 \%$ women) have been screened with mean age of 58 yrs (24-84y). 65 (48\%) have RA and remaining with connective tissue diseases. Median disease duration is $10 \mathrm{yr}(0.75-30$ yrs) and median drug exposure is $10 \mathrm{yr}(0.4-27 \mathrm{yr})$. Three doses of $\mathrm{HCQ}$ are prescribed: $200 \mathrm{mg}$ daily (53\%), 300mg daily (13\%) and $400 \mathrm{mg}$ daily (34\%). Ten (7.3\%) patients were found to have abnormal results. Three were consistent with HCQ toxicity pattern and one with likely toxicity. Two of them had already developed severe sight loss. HCQ was discontinued in all these cases. Six had other incidental anomalies requiring further input.

Conclusion: Hydroxychloroquine is used increasingly in the treatment of autoimmune diseases with emerging role in oncology. It has a favourable safety and tolerability profile with survival benefit demonstrated in SLE. In the UK, it's adoption has been particularly high owing to the requirement of trialling two DMARDs prior to being eligible for biologic therapy in RA and PsA. In the absence of modern retinal imaging techniques, HCQ toxicity was perhaps underestimated and hence older guidelines did not emphasise strict monitoring practice. Our preliminary data, in line with published evidence, represents a greater public health problem than previously estimated. It is clear that implementing the new guidelines not only recognises hitherto undiagnosed drug toxicity but also identifies incidental significant eye pathology which puts pressure on healthcare resources and needs robust service planning. Rheumatologists need to be aware of the potential impact requiring informed discussion with patients and perhaps a fundamental shift in prescribing behaviour to avoid this rapidly developing health concern

Disclosure of Interests: Amr Moharram: None declared, Freddy Raj: None declared, Jose Maya: None declared, Ranjit Sandhu: None declared, Muhammad Khurram Nisar Grant/research support from: Muhammad Nisar undertakes clinical trials and received support (including attendance at conferences, speaker fees and honoraria) from Roche, Chugai, MSD, Abbvie, Pfizer, BMS, Celgene, Novartis and UCB

, Consultant of: Muhammad Nisar undertakes clinical trials and received support (including attendance at conferences, speaker fees and honoraria) from Roche, Chugai, MSD, Abbvie, Pfizer, BMS, Celgene, Novartis and UCB

, Speakers bureau: Muhammad Nisar undertakes clinical trials and received support (including attendance at conferences, speaker fees and honoraria) from Roche, Chugai, MSD, Abbvie, Pfizer, BMS, Celgene, Novartis and UCB DOI: 10.1136/annrheumdis-2020-eular.2025

\section{AB1183 TERIPARATIDE SWITCH TO BIOSIMILAR - IS IT COST EFFECTIVE?}

T. Akram¹, M. Siddique ${ }^{1}$, H. A. Javed ${ }^{1}$, J. Begum ${ }^{1}$, J. Fourmy ${ }^{1}$, M. K. Nisar ${ }^{1}$. ${ }^{1}$ Luton and Dunstable University Hospital, Luton, United Kingdom

Background: Teriparatide is an effective treatment option for osteoporosis however NICE restricts its use to patients with high disease burden. This was based on cost effectiveness evaluation of the originator (Forsteo®) and would be different for recently introduced generic preparation.

Objectives: We wished to evaluate the current prescribing behaviour prior to a potential switch to generic version and associated cost savings.

Methods: All patients prescribed Teriparatide since the commencement of specialist osteoporosis service in Aug 2014 at our University teaching hospital covering 350,000 population were included. Data was extracted from electronic database with full access to demographics, population characteristics, disease parameters and medication history.

Results: 113 patients were prescribed Teriparatide over five years. Mean age of participants was 76 yrs (53-96). They had on average three comorbidities (0-8) with most common being hypertension $(n=44,38.9 \%)$ and inflammatory arthritis $(n=21,18.5 \%)$. Sixteen $(14.1 \%)$ individuals had concurrent corticosteroids. Median number of fractures prior to therapy were four (0-12). Prior treatments included oral therapy $(n=90,79.6 \%)$, IV zoledronate $(n=22,19.4 \%)$ and denosumab $(n=19,16.8 \%) .66(58.4 \%)$ of patients only had one prior bone active medication. Mean duration of prior therapy was 62.4 months (9-192 months). 17 $(15.0 \%)$ patients had chronic kidney disease with lowest eGFR of 38.41 (36.2\%) had Vit D level between 40-75 nmol/L. Median T score was -3.8 (-2.1 - -6.0) which improved to $-3.4(-2.9-3.9)$ after two years.

Conclusion: Our real-world study shows that teriparatide is used predominantly in complex, multi-morbid older individuals with several prior fractures. Despite that teriparatide remains effective for a wide range of individuals including those with inflammatory arthritis and/or concurrent steroid use. Neither moderate CKD nor mild Vit D insufficiency seems to impact its efficacy.
This is in line with recent meta-analysis of real life teriparatide use in complex osteoporosis with multimorbidity. Our study should enhance clinicians' confidence in its prescribing. It's notable that the use is higher than current estimates based on NICE cost effectiveness analysis for eligibility of teriparatide. Instead of annual predicted use of $4.8 / 100,000$ population, it was prescribed to $6.4 / 100,000$. This could potentially have a cost impact however the introduction of a generic version would mitigate against it. We calculated our savings to be over $£ 125,000$ if all patients were switched. These savings at national level would hopefully improve access to a wider patient cohort and perhaps allow earlier use in the treatment paradigm.

Disclosure of Interests: Tahreem Akram: None declared, Maham Siddique: None declared, Hafiz A. Javed: None declared, Julie Begum: None declared, Joanne Fourmy: None declared, Muhammad Khurram Nisar Grant/research support from: Muhammad Nisar undertakes clinical trials and received support (including attendance at conferences, speaker fees and honoraria) from Roche, Chugai, MSD, Abbvie, Pfizer, BMS, Celgene, Novartis and UCB

, Consultant of: Muhammad Nisar undertakes clinical trials and received support (including attendance at conferences, speaker fees and honoraria) from Roche, Chugai, MSD, Abbvie, Pfizer, BMS, Celgene, Novartis and UCB

Speakers bureau: Muhammad Nisar undertakes clinical trials and received support (including attendance at conferences, speaker fees and honoraria) from Roche, Chugai, MSD, Abbvie, Pfizer, BMS, Celgene, Novartis and UCB DOI: 10.1136/annrheumdis-2020-eular.1970

\section{$\mathrm{AB} 1184$ BURDEN OF DISEASE AND DIRECT HEALTH CARE COSTS FOR PATIENTS WITH SYSTEMIC LUPUS ERYTHEMATOSUS IN WESTERN AUSTRALIA}

W. Raymond ${ }^{1}$, G. Ngo ${ }^{1}$, M. Ognjenovic ${ }^{1}$, I. Li $^{2}$, P. Cheah $^{3}$, A. Chakera ${ }^{4}$, A. Mclean-Tooke ${ }^{5,6}$, J. " Nossent ${ }^{1,3}$ on behalf of Perth Working Party on SLE. ${ }^{1}$ The University of Western Australia, Rheumatology Section, School of Medicine, Crawley, Australia; ${ }^{2}$ The University of Western Australia, School of Population \& Global Health, Crawley, Australia; ${ }^{3}$ Sir Charles Gairdner Hospital, Rheumatology, Nedlands, Australia; ${ }^{4}$ The University of Western Australia, Nephrology, School of Medicine, Crawley, Australia; ${ }^{5}$ Sir Charles Gairdner Hospital, Immunology, Nedlands, Australia; ${ }^{6}$ PathWest, Laboratory Medicine, Nedlands, Australia

Background: Systemic Lupus Erythematosus (SLE) is a chronic multiorgan disease with an unpredictable disease course, which requires monitoring for disease activity, treatment efficacy and comorbidity. Data on the healthcare utilization and cost of SLE, especially from Australia are scarce.

Objectives: To determine the healthcare utilisation and estimated costs of inpatient admissions (IP), emergency (ED) and outpatient (OPD) hospital visits and investigations for SLE patients in Western Australia (WA).

Methods: This is a longitudinal cohort study of SLE patients seen at a metropolitan public hospital, with $\geq 6$ months of follow-up ( $n=179,95 \%$ female; baseline age $36.2 \pm 15.2$ years). Electronic medical records provided data on OPD, ED and IP visits, and investigations conducted at public hospitals from January 2000 - December 2019. Direct healthcare costs were estimated from public hospital expenditure aggregates in FY2018/19.

Results: During a median observation period of 11.0 years (IQR 7.4, 13.5), SLE patients required 13,320 OPD visits for a median of 5.3 (IQR 3.0,9.3) appointments per annum. The majority of OPD visits were with Rheumatology $(n=1,986$, $14.9 \%)$, Immunology $(n=1,527,11.5 \%)$, and allied health services $(n=1,952$, $14.7 \%)$, followed by Ophthalmology $(n=1,385,10.4 \%)$, maternal \& fetal health $(n=873,6.6 \%)$ and Renal medicine $(n=844,6.3 \%)$. In total 143 patients $(79.9 \%)$ attended ED on average of 3 times (IQR 2, 7; ED visit rate $44.0(95 \% \mathrm{Cl} 41.0$, 47.0) per 100 person years. Overall, 125 patients (69.8\%) were hospitalised at average 3 times (IQR 2, 6), with a mean LOS of 5 days (IQR 3, 12) for an IP rate of 37.6 per 100 patient years $(95 \% \mathrm{Cl} 34.8,40.5)$. Only $12.8 \%$ of patients did not attend ED or IP in the public health care system. A total of 367,067 laboratory investigations were performed (median nr. of tests per patient $205( \pm 290)$ per year) across fields of haematology/biochemistry (89\%), immunology (5\%), microbiology $(4.5 \%)$ and histopathology $(<1 \%)$. Minimum estimates for direct health care cost during the study period were 25.4 million AUD (IP $11 \mathrm{~m}$, OPD $6.3 \mathrm{~m}$, ED $0.9 \mathrm{~m}$ and investigations $9.1 \mathrm{~m}$ ) for a crude annual cost of 14,088 AUD per patient.

Conclusion: SLE patients have extensive healthcare utilization across a range of outpatient and inpatient services. The main direct costs for this multidisciplinary health care provision are for disease monitoring and in-hospital treatment Based on these conservative cost estimates to which medication cost need to be added, total costs for SLE care in WA are projected to be significantly higher than reported from Europe. 